\title{
Filigrane
}

Écoutes psychanalytiques

\section{En mouvement. Entretien avec Catherine Chabert}

\section{Louise Mercier}

Volume 25, numéro 1, printemps 2016

Actes de la journée clinique de la SPQ (Catherine Chabert)

URI : https://id.erudit.org/iderudit/1037379ar

DOI : https://doi.org/10.7202/1037379ar

Aller au sommaire du numéro

Éditeur(s)

Revue Santé mentale au Québec

ISSN

1192-1412 (imprimé)

1911-4656 (numérique)

Découvrir la revue

Citer cet article

Mercier, L. (2016). En mouvement. Entretien avec Catherine Chabert. Filigrane, 25(1), 131-143. https://doi.org/10.7202/1037379ar d'utilisation que vous pouvez consulter en ligne.

https://apropos.erudit.org/fr/usagers/politique-dutilisation/ 


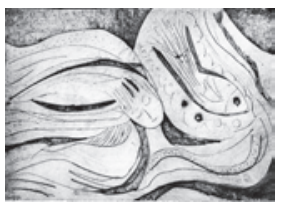

\title{
En mouvement. Entretien avec Catherine Chabert
}

\author{
Louise Mercier ${ }^{1}$
}

Louise Mercier: Bonjour Catherine Chabert, et merci d'avoir accepté de vous entretenir avec moi, à l'occasion de ce séjour à Québec. J'aimerais commencer par une brève présentation. Vous êtes professeure émérite en psychologie clinique à l'Institut de psychologie de l'Université Paris Descartes, codirectrice avec Jean-Claude Rolland des Libres Cahiers pour la psychanalyse. Vous êtes l'auteure de nombreux livres et articles. Vous faites aussi de l'enseignement à l'université. Vous avez une pratique clinique à la fois en bureau privé et à l'hôpital. J'aimerais que vous nous parliez, avant d'aborder votre travail d'écriture, de votre parcours, de ce qui vous a amenée à la psychanalyse.

Catherine Chabert: Alors, quelques petites rectifications parce que le temps passe et les choses changent. Effectivement, je suis professeure émérite, c'està-dire que j'ai arrêté mes activités universitaires il y a deux ans. C'est vrai que j'ai longtemps travaillé à la fois à l'université, en libéral, et à l'Institut Mutualiste Montsouris (un service de psychiatrie de l'adolescent et des jeunes adultes). J'y ai vraiment trouvé une grande source d'intérêt et d'expérience. J'aurai l'occasion d'y revenir. Par ailleurs, la publication des Libres Cahiers pour la psychanalyse que j'ai dirigée pendant plus de 15 ans avec Jean-Claude Rolland s'est terminée l'année dernière. Donc, il y a des choses qui appartiennent au passé et d'autres, au présent et davantage tournées vers l'avenir.

Pour répondre à votre question: je ne suis pas venue tout de suite à la psychanalyse. D'abord, j'ai une formation littéraire et j'ai fait très brièvement des études de philosophie. J'ai obtenu une licence de philosophie. Puis je me suis engagée dans des études de psychologie. J'habitais en Provence, dans le sud de la France, à une période où la psychanalyse commençait tout juste à poindre dans les universités de la région. Dans l'université où j'étais étudiante et où je faisais beaucoup de psychologie expérimentale et de psychologie sociale - ce qui était tout de même dans mon cursus j'ai rencontré René Kaës qui a été mon professeur. Je suis alors devenue 
extrêmement intéressée par la dynamique de groupe; il m’a donc engagée à titre d'observatrice dans ceux-ci. C'est par lui, par ses enseignements que j'ai décidé de venir à Paris parce que j'avais envie de faire du psychodrame. Mais à cette époque, je n'avais même pas 20 ans, et du côté de la psychanalyse, j'avais des réserves. La psychanalyse m’intéressait en tant que telle bien sûr, mais même devenir psychologue clinicienne, je trouvais que c'était trop de responsabilités par rapport aux autres, par rapport aux patients. Bref, j'avais énormément de résistances qui ont très vite cédé au cours de ma rencontre avec Didier Anzieu. Je voulais m’inscrire dans son université. Je lui ai bien expliqué avec beaucoup de «rationalité» mon projet: je voulais faire de la psychologie sociale clinique, mais surtout pas de la psychopathologie. Et puis je suis sortie de son bureau inscrite en psychopathologie et dès lors, ce fut pour moi une espèce d'emballement, de passion. Mes profs, ils étaient jeunes... À l'époque, j'ai rencontré d'abord Didier Anzieu qui a beaucoup compté dans ma vie, Daniel Widlöcher qui faisait de la psychopathologie de l'enfant, Roger Dorey...

Puis, j'ai fait du psychodrame analytique pendant un an. C'était une période où les enseignements se faisaient vraiment dans une dimension extrêmement humaine. Il y avait ce qu'on appelait des "présentations de malades», devant quinze étudiants, par Roger Dorey ou Daniel Widlöcher. Évidemment, en étant engagée avec eux comme enseignants, j'étais extrêmement intéressée par la psychanalyse et j'ai eu du plaisir à travailler. C'est pour cela que je suis restée très fidèle à l'idée que la place de la psychanalyse à l'université était importante puisqu'elle permettait une sensibilisation des étudiants, et plus encore, qu'elle pouvait y laisser une marque décisive. Pourtant, dans les années 70, à une période où la psychanalyse occupait le devant de la scène à la fois sur le plan intellectuel et culturel, ce n'était pas très bien vu d'être universitaire dans le milieu analytique: on était pour la psychanalyse pure, pas hors les murs. Il y a eu beaucoup de débats à cette époque entre les analystes: ceux qui travaillaient à l'université comme Laplanche, Fedida et Anzieu, et ceux qui ne voulaient pas y aller. Quelqu'un comme Pontalis a été longtemps très réservé par rapport à une implication universitaire. Aujourd'hui, ça parait étrange, mais c'était comme ça.

Je suis entrée à Paris-V, Paris Descartes (à l'époque ça s'appelait ParisSorbonne), pour obtenir mon diplôme de psychopathologie et puis c'est là quej'ai découvert, en rencontrant Nina Rausch de Traubenberg, les méthodes projectives et surtout l'interprétation psychanalytique des épreuves projectives. Comme j'étais très jeune (j'avais obtenu mon baccalauréat rapidement) 
et que j'aimais les études - même si j'étais un peu paresseuse à l'époque, beaucoup plus que maintenant —, quand j'ai eu mon diplôme, il m'a semblé impossible de ne pas poursuivre des études. J'ai donc été voir Widlöcher pour faire une Thèse de Troisième Cycle en psychologie clinique et en psychopathologie. Je lui ai dit — je me souviens, il avait ri très discrètement d'ailleurs... encore une fois j'étais très jeune - : «je voudrais faire une thèse sur le surmoi !» Alors, il avait souri et il m’avait répondu: «Oh! Ça, c’est une question qu'il est difficile d'aborder de front.» Mais quand même, il m'avait prêté un rapport du Congrès des psychanalystes de langue romane consacré au surmoi. Je me souviens combien j'avais travaillé dessus alors que j'avais une très faible culture en ce qui concernait les écrits de Freud. Et puis, quasiment tout de suite, il m'a proposé un poste d'assistante à l'université. C'était un statut intéressant, c'est-à-dire que, pour le temps de la thèse, on assurait un certain nombre d'heures de cours.

LM: Vous étiez vraiment bien entourée.

CC: Ah oui, j'ai fait des rencontres formidables.

LM: Je voulais aborder vos influences, mais là vous en parlez. J'avais en tête Freud qui est au cœur de votre travail. On pourrait y revenir... Pontalis aussi.

CC: Pontalis, je l'ai connu beaucoup plus tard quand je suis rentrée à l'Association Psychanalytiques de France.

LM: On pourra y revenir aussi... Mais dans votre écriture, il y a quelque chose de l'écriture de Pontalis. Il y a tellement de richesse dans tout ce que vous nous avez apporté hier ${ }^{2}$, dans tous vos écrits... Je voudrais revenir sur certaines élaborations que vous avez faites. Vous me resituerez dans le temps, mais dans les 15 ou 20 dernières années, vous vous êtes beaucoup attardée aux problématiques narcissiques identitaires et à tout ce qui est de l'ordre de la mélancolie... J'aimerais rapporter ici des propos que vous avez tenus, qui font bien le lien entre le livre Féminin mélancolique, écrit en 2003, et l'un de vos derniers livres, L'amour de la différence, qui a été le sujet de notre travail hier. Tout au long de vos écrits, vous nous dites: «je me suis d'abord laissée emporter par les excès narcissiques de la mélancolie en y cherchant d'autres formes de la séduction et du masochisme.» Dans Féminin mélancolique, ça a été plus ce que vous appelez la "première logique», davantage dans la logique du même ou de l'indifférenciation en lien avec la deuxième topique... jusqu'à L'amour de la différence où vous nous parlez d'une deuxième logique liée celle-ci à la première topique. Vous nous dites: «je me suis attachée cette fois-ci à une autre logique. Celle de la différence qui soutient et 
affirme au-delà de l'altérité l'existence et la reconnaissance d'objets internes pris dans le réseau de la sexualité quel qu'en soit le registre.» À l'intérieur de tout ça, on sent que l'CEdipe devient votre voie privilégiée d'élaboration. Vous pouvez nous parler de ce parcours dans vos écrits?

CC: Alors... en vérité, cette question du féminin mélancolique est le produit d'une longue expérience de mon travail d'analyste à l'Institut Mutualiste Montsouris où je suis entrée en 1975, au moment où je commençais ma formation analytique. C'est un service de Psychiatrie de l'adolescent et du jeune adulte, et c'est un service dans lequel on recevait, et où l'on continue d'ailleurs de recevoir, beaucoup de jeunes filles qui présentent de graves troubles des conduites alimentaires et de tentatives de suicide. J'ai aussi travaillé à l'hôpital de jour avec des patients psychotiques et des patients borderline sévères.

La question du narcissisme et de la sexualité se pose de manière particulièrement aiguë à différents moments de la vie, même si elle nous accompagne du début à la fin; au moment de l'adolescence, elle est particulièrement vive. Je me suis retrouvée avec ces jeunes filles qui présentaient des conduites autodestructrices visant préférentiellement leur corps; mais ce n'est pas simplement le corps, c'est le corps sexué qui était visé. C'était leur corps de femme, leur corps de fille qui était attaqué. Cela montre bien que la question de l'identité sexuelle demeure, même lorsqu'il y a une souffrance ou une douleur psychique qui s'attache à d'autres problématiques. Plus particulièrement chez ces jeunes filles, il y avait cette attaque du corps très autodestructrice, mais il y avait aussi une autre dimension dans leur symptomatologie qui m’a beaucoup retenue, à savoir la dimension compulsive et le recours à l'acte. On voyait bien au décours des traitements - ce qui est assez connu et qui s'est dégagé assez rapidement au niveau de la prise en charge de ces jeunes filles - que ces conduites agies et compulsives ont comme visée l'évitement de la douleur psychique, et en particulier de la dépression et la souffrance psychique attachée à la perte d'objet. Il y avait donc cette conjonction du corps de femme attaqué, associé à la prégnance de la question du sexuel, et tout aussi fortement intriquée, la question de la perte et l'extrême difficulté à se séparer et en particulier à se séparer de la figure maternelle.

C'est là que se sont articulées pour moi les questions du narcissisme et de la mélancolie. Et c'est aux traitements analytiques que je dois cette prise de connaissance; il y avait une tendance à penser en psychopathologie analytique — et je caricature — de façon assez ciblée, c'est-à-dire que la 
névrose était une problématique sexuelle et de castration, les fonctionnements limites relevaient de difficultés au niveau de la perte d'objet et de la dépression, et les fonctionnements psychotiques étaient liés aux angoisses de morcellement et à la perte de contact avec la réalité. Et puis, étaient repérées des configurations conflictuelles attachées à ces catégories et on avait tendance à penser que le complexe d'CEdipe, qu'on entendait dans ses formes relativement structurantes, concernait seulement les névroses et pas les autres organisations psychopathologiques considérées comme pré-oedipiennes. Ce que j'ai découvert avec ces jeunes patientes, c'est une problématique oedipienne flamboyante. Tellement flamboyante qu'elle nécessitait des recours défensifs drastiques, coûteux et finalement délétères. L'objet principal de Féminin mélancolique, même si plusieurs autres thématiques y apparaissent, est cette idée des fantasmes de séduction dans leur version mélancolique. Dans la version classique, hystérique, du fantasme de séduction, c'est l'autre, c'est l'adulte, c'est le père qui est accusé d'être le séducteur, mais chez ces filles-là, ce qui apparaissait était quelque chose d'autre: c'était elles qui étaient coupables, c'était leur corps de femme qui était excitant, c'était elles qui étaient activement séductrices. Et donc, elles étaient à la fois criminelles et bourreaux. Au fond, c'est ça le noyau de Féminin mélancolique. Du coup, comme je suis une lectrice passionnée de Freud, je suis revenue aux textes et j'ai décortiqué Deuil et mélancolie pour essayer d'avancer de ce côtélà et en particulier pour dégager une forme d'identification mélancolique qui va au-delà des identifications hystériques et au-delà des identifications narcissiques.

LM: On sent quand même dans tout ce parcours, quand on vous lit, qu'il y a toute une portée philosophique, en lien avec les écoles de psychanalyse un peu partout qui accordent une grande priorité à l'objet primaire. Vous, vous ne niez pas ça, loin de là, vous y retournez. Mais on sent ce besoin de faire de l'Edipe l'une des voies d'élaboration privilégiée du travail... comme quelque chose d'important. Qu'on revienne à l'CEdipe aussi...

CC: Oui, tout à fait. C'est l'idée finalement qu'il y a un horizon oedipien ou des sources oedipiennes qui sont là de toute manière au niveau de cette configuration. Pour moi, le risque de la psychanalyse à certains moments est d'abandonner la question de la sexualité et aussi de l'CEdipe, qui constitue une référence primordiale parce qu'elle contient à la fois l'ambivalence entre l'amour et la haine, la question des identifications et la référence au masculin et au féminin, la conflictualité entre les désirs et les interdits, et les choix d'objet. C'est dire que l'Eedipe est une configuration qui condense 
plusieurs courants de problématiques. Ce qui m'intéresse n'est pas tellement de poser l'CEdipe comme une représentation/but ou une structure coiffante, ce sont les différentes modalités de construction de l'Edipe qui peuvent être extrêmement variables d'un individu à un autre. Ce que je n'utilise en aucune manière sur un mode diagnostique: ce n'est pas à partir du repérage de l'CEdipe que l'on va dire qu'on est ou non dans la névrose et en tirer des conséquences.

Peut-être que j'ai été étonnée par le mot que vous avez utilisé: philosophie. Il me semble que dans le mouvement même de la cure, dans la méthode analytique, ne pas se replier uniquement sur le duel, sur le fusionnel, sur des commencements qui seraient pris dans une espèce d'entité toute-puissante... Ce n'est pas que ça n'existe pas, mais qu'est-ce qu'on fait pour s'en dégager? Quelles sont les voies de construction? Quel dynamisme peut-on essayer d'en dégager?

LM: L'importance de la régression...

CC: Exactement. Mais ce n'est pas du tout la même chose de penser qu'on est pris comme ça, englué massivement dans une relation originaire, et penser l'analyse sous forme de régression. Je pense en effet qu'il y a des moments de l'analyse qui sont extrêmement pénibles et difficiles, où la régression l'emporte. Et on est pris dans ces mouvements de confusion, d'emballement, de mélancolie autant pour l'analyste que pour le patient. Enfin, je ne récuse pas le caractère extrêmement essentiel des commencements de la vie psychique, mais il y a quelque chose qui m'importe et où la philosophie de la psychanalyse me semble utile: les choses ne sont pas définitivement mises en place dès le début. Dans les perspectives trop biologisantes, on peut avoir l'impression que c'est comme ça: il y a un donné. On pourrait penser aussi, de manière analogique, que dans certaines positions psychiatriques ou psychopathologiques, puisque que ça s'est passé comme ça dans les débuts, ça va rester identique, ça ne bougera pas. Dans les perspectives de la psychanalyse, on peut penser (ou croire) que les potentialités de changement existent même si elles sont très différentes, plus ou moins mobilisables par et dans le transfert; plus ou moins ouvertes selon les individus. Au fond, pour moi, l'analyse est une manière de ne pas croire au destin.

LM: De remettre en mouvement.

CC: De remettre les choses en mouvement. De penser qu'effectivement il peut y avoir une mobilisation, grâce au transfert, des potentialités de changement, et qu'on arrive comme ça à un peu plus de liberté. Comme je pense que la relation duelle peut être éminemment engluante et enlisante, je 
trouve qu'il est important, sans excès de suggestion évidemment, de penser les choses dans la différence et en particulier dans la différence des sexes. Même si cela n'exclut pas la logique de la mélancolie qui, je pense, est extrêmement présente.

LM: On sent l'enchevêtrement dans votre livre.

CC: C'est ça, c'est un enchevêtrement pour moi.

LM: Et c'est agréable dans ce sens-là. C'est de l'ouverture. On a l'impression quand on vous lit d'être toujours au cœur de l'inconscient et surtout, dans la chair de l'inconscient, au plus près du corps. C'est très vivifiant, je dirais. Pour faire un lien avec le «féminin pur» de Winnicott, que vous abordez peut-être dans Féminin mélancolique, mais certainement dans L'amour de la différence... On sait que le féminin est central dans votre travail. Vous nous parlez d'un féminin qui est inscrit dans le registre de l'Edipe, mais d'un féminin qui plonge aussi ses racines dans les arcanes de l'angoisse de perte de l'amour de l'objet. Je reviens à vos propos parce que c'est très dense, je veux être au plus près de ce que vous nous amenez... «Dans ce double mouvement, la bisexualité — le masculin et le féminin pour les deux sexes déjà là en partant est fortement engagée.» Mais on a l'impression aussi que ce féminin-là serait antérieur en quelque sorte au féminin-masculin de la bisexualité quand vous nous dites: «Ce féminin-là, point de sédimentation essentiel de la bisexualité et des identifications, laisserait une empreinte indélébile, témoignant d'une séparation toujours impossible ou imparfaite d'avec la mère et marquant à jamais la condition des Hommes.» C'est une question qui est toujours là quand je vous lis: j'ai l'impression qu'il y a un féminin qui est là, qui vient vraiment de cette première relation, et qui est différent de la bisexualité. Est-ce que ça fait sens pour vous?

CC: Oui, j'accorde une grande importance à l'identification dans la construction psychique. Alors, c'est vrai que la question de la relation à l'objet primaire me parait importante, mais dans le passage que vous citez, ce que j'avais essayé de dégager est l'idée qu'au bout du compte, cette entité mèreenfant des commencements de la vie laisse une empreinte en termes d'une identification à cette mère-là. Je ne sais pas si je le dirais de la même façon aujourd'hui d'ailleurs, mais pourquoi ne pas y revenir parce que cela faisait partie aussi de mes convictions. Encore une fois, c'est en termes d'identification, dans une espèce de prise de la mère et de l'enfant que j'inscrirais ces empreintes originaires. Et pas encore dans la question des modalités des relations entre la mère et le bébé. Le soubassement, le tissu même de ces modalités d'engagement dans la vie entre l'objet et le moi, même s'il est 
très embryonnaire à ce moment-là, ce serait ce système d'identifications qui garderait cette empreinte.

LM: Sur laquelle la bisexualité serait en quelque sorte reprise.

CC: Exactement. Au fond, si on se réfère à Laplanche par exemple et à la question des signifiants ou des messages énigmatiques, quand je dis que le masculin et le féminin sont là dès les origines, c'est parce qu'ils sont psychiquement là dans la mère. Il y a cette empreinte des identifications des débuts de la vie qui est présente et je m'interroge... Mais pour l'instant, comment le savoir? Est-ce que d'ailleurs on arrivera à le savoir vraiment? Dans cette identification à la mère, il doit bien y avoir des traces de l'autre sexe. Je ne sais pas comment, mais je pense qu'à l'origine, il y a cette identification-là, ce qui donne ensuite, de manière peut-être un peu plus construite, l'identification narcissique.

LM: Et qu'est-ce qui vous a amenée à l'intérieur de tout ça, à retourner au «féminin pur» de Winnicott?

CC: Le «féminin pur» de Winnicott est quelque chose que j'ai d'abord admis... J'aime beaucoup Winnicott; je trouve qu'il est très important de le lire, parce qu'il a apporté énormément d'idées d'une très grande subtilité et d'une très grande puissance sur le plan de la clinique, mais aussi de la métapsychologie. Et je trouve (ce qui arrive pour tous les grands, et qui est arrivé aussi pour Freud) qu'on en est venu finalement à banaliser Winnicott et à en faire un théoricien de la première ou de la petite enfance. La plus belle illustration est la commercialisation des objets transitionnels. On oublie ainsi qu'il a énormément réfléchi, travaillé et écrit sur le plan théorique; on oublie qu'il a construit une théorie en rapport avec celle de Freud, mais avec des éléments de divergence à certains moments - une divergence qu'il assume, d'ailleurs. Par exemple, il propose toute une construction théorique autour de la psychose.

LM: Il revient à Freud tout en s'en distinguant...

CC: Absolument. Et il se sert dans ses définitions des concepts freudiens. Alors, ce clivage entre les éléments masculins et les éléments féminins: peutêtre, pourquoi pas, je ne sais pas... J'ai commencé par lire, par travailler et par utiliser le «féminin pur» dans des communications, des exposés ou des articles. Puis, éventuellement, je suis revenue au texte, et j'ai été gênée par ce qu'il y déclare, à savoir que ce «féminin pur» serait décontaminé, défait de toute dimension pulsionnelle. Et là, tout à coup, il y a quelque chose qui m'a gênée, dans la mesure où pour moi dès les débuts de la vie, la pulsion est là, le pulsionnel est là; c'est ça la vie. 
Ou alors je n'ai pas bien compris et pour Winnicott, il s'agit d'une représentation fantasmatique de mère idéale, pure, décontaminée et cetera. Il faut peutêtre retourner au texte... Si c'est un produit fantasmatique, pourquoi pas?

LM: Justement, vous avez repris le «féminin pur» dans vos cures, en parlant plutôt d'une production fantasmatique des analysants.

CC: Tout à fait. Mais comment faire la différence avec une représentation maternelle absolument idéalisée? Parce qu'il ne parle pas de toute-puissance à propos du «féminin pur»...

LM: Son article est vraiment complexe.

CC: Oui, il faut que je le relise encore.

LM: C'est intéressant quand vous faites le parallèle avec ce fantasme que vos analysants, hommes ou femmes, peuvent développer d'une mère quasi désincarnée.

CC: Tout à fait. Désexualisée. Quand ça apparaît dans des cures d'adultes, c'est vraiment une déformation, un produit...

LM: En lien avec la scène primitive... parce que vous élaborez beaucoup là-dessus aussi.

CC: Voilà. On retrouve quand même le refus d'envisager la femme derrière la mère: la femme sexuelle, désirante...

LM: Avant qu'on termine, j'aimerais parler de ce qui s'en vient. De ce qui est là, mais dont on a moins entendu parler. Vous avez publié un nouveau livre. Je vous entendais parler de toute votre expérience avec les adolescentes au début de votre carrière, on sent que c'est une expérience qui a été très riche, très chargée, je ne sais pas si ça a duré longtemps...

CC: Oui... J'ai travaillé à l'Institut Mutualiste Montsouris pendant une trentaine d'années.

LM: Mais c'est intéressant de voir que vous revenez sur l'adolescence, aujourd'hui après toutes ces années, dans votre nouveau livre La jeune fille et le psychanalyste. Est-ce que vous pourriez nous en parler?

CC: D'abord, il est vrai que je me suis beaucoup intéressée à l'adolescence et à ce passage. Je m'intéresse aux passages, aux mouvements.

LM: Là où ça bouge...

CC: Où ça bouge et où il y a des possibilités... mais aussi des prises de risques. Toutes ces périodes de passage ne vont pas inéluctablement donner lieu à une construction favorable. Dans les traitements analytiques de ces jeunes adolescentes, il y avait des risques et d'ailleurs ça n'a pas toujours fonctionné. Il y avait aussi des évolutions défavorables, négatives, inquiétantes... Ce sont des prises en charge très périlleuses. 
Mais j'aimais beaucoup travailler à l'hôpital parce que justement, ces patientes ne pouvaient pas être reçues en privé.

LM: Le risque de bascule était trop fort en quelque sorte.

CC: Voilà. Il m'est arrivé, à la sortie d'une séance de psychothérapie, d'appeler le psychiatre de garde parce que la patiente me disait qu'elle allait se suicider l'après-midi même... dans une espèce de complicité séductrice avec laquelle il était impossible de fonctionner.

J'ai beaucoup travaillé à l'hôpital. Alors, dans mon dernier livre, La jeune fille et le psychanalyste, j'ai repris un certain nombre de textes que j'avais précédemment communiqués ou publiés à partir de mon travail avec ces jeunes filles. Et pas seulement à l'hôpital. Je suis partie il y a une dizaine d'années de l'hôpital pour différentes raisons, en particulier parce que j'avais des charges universitaires énormes et que j'avais décidé de travailler davantage dans mon cabinet. Je continue à recevoir des jeunes femmes en traitement analytique, pour la plupart avec des pathologies moins sévères, quoique j'en reçoive quelques-unes plus sévèrement atteintes. Donc, cet ouvrage, ça faisait longtemps que je l'avais en tête. Les éditions Dunod m'avaient proposé de reprendre un ouvrage pour leur collection «Psychisme» créée par Didier Anzieu. Curieusement, j'ai mis beaucoup de temps alors qu'il y avait beaucoup de choses qui étaient rédigées. C'est difficile de reprendre des articles pour en faire un livre; c'est beaucoup plus difficile qu'on ne l'imagine. Et puis, finalement, j'ai décidé de m'y mettre pour de bon. Ce livre est paru en juin dernier, j'ai dû le rendre à l'automne précédent. Je me suis vraiment résolue à m'y mettre avec une espèce de résistance: j'étais inquiète parce que c'est un livre qui est extrêmement clinique, et il y a une articulation clinique et métapsychologique qui m'importe énormément. C'est aussi une manière de travailler et un mode d'écriture particulier, parce que lorsqu'il y a énormément de clinique, on abandonne le style parfois un peu austère et condensé de la métapsychologie. En fait, dans chacun des chapitres de ce livre, une problématique se dégage, mais il y a une histoire clinique de traitement de jeunes filles, avec des fragments de cures. Le moteur de ce livre est le transfert. LM: Oui, on le sent toujours présent dans votre écriture.

CC: Et je pense qu'au niveau de l'écriture, je me suis un peu «lâchée » : c'està-dire que dans les récits cliniques, il y a une dimension littéraire.

LM: Vous les avez repris, donc?

CC: Je les ai retravaillés... certains moins que d'autres, parce que la forme me convenait. Dunod ne m'a pas du tout consultée, mais quand j'ai reçu la couverture, j'étais enchantée par cette jeune fille en robe bleue, un peu à 
l'ancienne. Elle m'a rappelé un de mes souvenirs. Moi, j'étais une petite fille à grands cheveux et j'avais une robe en velours bleu comme ça.

LM: C'était vous finalement! (rires...)

CC: C'est drôle, ça vient bien dire la chose: ce qui est mobilisé aussi chez l'analyste, la petite fille, la jeune fille et la femme. C'est bien pour ça que j'ai eu la coquetterie d'appeler ce livre La jeune fille et LE psychanalyste.

LM: C'était intéressant de vous voir revenir à l'adolescence. Quand vous parlez de passage dans la vie, qui sera repris plus tard aussi quand même, il y a ce mouvement-là...

CC: Exactement, pour moi c'était une condition pour la publication. En fait, ce sont des patientes que j'ai rencontrées il y a longtemps. C'était essentiel.

LM: Oui, le temps est important pour la publication. Vous avez travaillé sur toute la dimension mélancolique. Ce qui s'en vient ou ce qui a été déposé dernièrement, c'est votre article sur l'amour maniaque. Il y a quelque chose là de vraiment intéressant: vous vous inspirez de Nathalie Zaltzman, vous vous inspirez de la pulsion anarchique que vous abordez d'une manière un peu différente. J'aimerais que vous m'en parliez.

CC: J'ai lu tardivement dans ces dernières années les écrits de Nathalie Zaltzman. C'est quelqu'un d'extrêmement ferme; elle est très déclarative et c'est très intéressant qu'elle ait des positions aussi fermes. J'ai trouvé des idées et surtout des formulations de choses que je pouvais penser et que peut-être, je n'osais pas penser. Par exemple, tous ses développements sur la pulsion anarchiste et sur la pulsion de mort, c'est vrai que ça m’a confortée dans les positions que j'avais et que ça m'a donné une sorte d'argumentaire. Peut-être aussi l'appui sur une femme, mère et psychanalyste... Parce que ça faisait longtemps que je travaillais sur les pulsions de mort en considérant qu'on ne pouvait pas les réduire à des mouvements destructeurs. Effectivement, son écrit sur la pulsion anarchiste, je l'ai travaillé de très près. Nous avons organisé une journée scientifique avec Jacques André sur la psyché anarchiste et nous avons publié un petit livre collectif là-dessus.

C'est souvent les propositions qui nous font avancer. Françoise Coblence, qui dirige la Revue française de psychanalyse, m’a demandé de contribuer à un numéro sur la manie en s'appuyant sur le texte que j'avais écrit sur Don Juan - texte qui est dans mon livre dans le chapitre intitulé «L'inconstance». Comme ça faisait un moment, déjà avec «L'inconstance», mais aussi avec la question de la manie — au sens analytique et freudien du terme - , que j'étais revenue aux fragments freudiens sur la manie, je me suis engagée dans ce travail. 
LM: Vous étiez seule à ce moment-là?

CC: Oui. C'est quelque chose que j'ai fait l'hiver dernier toute seule; et j'ai décidé de travailler sur l'amour maniaque, comme moment de transfert, plutôt que sur la manie. Et c'est vrai que j'ai été très intéressée par l'article de Nathalie Zaltzman, un article qui m’a été donné par Laurence Kahn. Auparavant, je ne connaissais pas ce texte et ça a été un point de départ formidable parce que, là encore, elle récuse l'association trop convenue selon Melanie Klein entre position dépressive et défense maniaque. C'est la position kleinienne qu'elle critique et elle va chercher autre chose que ce binôme position dépressive/défense maniaque.

LM: Qu'elle trouve trop réducteur au fond.

$\mathrm{CC}: \ldots$ trop réducteur, pour cerner la question de la colère. J'ai réfléchi à ce que pouvait être cette colère. J'ai fait un parallèle entre la mélancolie et la manie, en particulier pour les dégager de leur connotation trop pathologique. De la même façon que je pense que dans n'importe quelle cure, il peut y avoir des moments mélancoliques, je réfléchis aux moments maniaques dans les cures...

LM: Nathalie Zaltzman parle de la colère, être fâché à mort... Vous, vous le reprenez différemment. Vous revenez à la relation, vous parlez de la qualification de la relation. Pourriez-vous nous en dire quelques mots?

CC: Oui, je parle de la qualification de la relation dans la mesure où au fond, parmi ce qui pourrait constituer les composantes de l'amour maniaque, il y a l'idée quand même d'une idéalisation et d'une représentation, une qualification de la relation à l'objet qui ne supporterait aucune entrave, aucune frustration, aucune atteinte. Donc, cet amour maniaque viendrait un peu comme une sorte de contre-investissement triomphant, certes soutenu par la colère, mais qui tendrait à abraser le motif de celle-ci. Mon idée est que la colère est liée, et c'est une idée ancienne que je ne formulais pas en termes de colère, qui a trait pour moi à l'expérience de ne pas être entendu, de ne pas être écouté. En particulier, c'est une scène paradigmatique, pas nécessairement une scène réelle, une scène où l'enfant serait en pleur et en chagrin et où il y aurait une disqualification: «Pourquoi tu pleures? Tu n'as aucune raison de pleurer!» Un refus de reconnaissance de la part de l'adulte, une impossibilité d'imaginer ou de reconnaitre le motif de la douleur psychique de l'enfant. Cette situation fantasmatique, je me suis demandée dans quelle mesure elle pouvait être traitée autrement que sur un mode mélancolique, c'est-à-dire avec un retournement contre soi de l'agressivité, un retournement qui chercherait à atteindre l'objet aussi, 
mais justement avec cette caractéristique de l'amour maniaque, soit un triomphe narcissique.

LM: C'est là que vous faites toute la distinction entre la séparation de l'objet qui est porteur de douleur alors que dans ce cas-là...

CC: Il n'y a pas de place.

LM: Il n'y a pas de place pour la douleur. Ça colmate tout ça.

CC: Maintenant je réfléchis à la manière dont ça peut se saisir dans la cure. Je pense qu'il y a des moments où l'analyste et le patient sont pris, sans qu'il s'agisse de manie, par cet amour maniaque. Je pense qu'il y a des moments de rencontre dans l'analyse où il y a une espèce de jubilation absolument formidable, parfois liée à une rencontre entre l'interprétation et avec ce que le patient veut dire. Au fond, la proposition de Françoise Coblence est un peu comme une interprétation pour moi. Elle, disant: «Tu as énormément travaillé sur la mélancolie. En même temps, tu amorces tout le temps la question de la manie. C'est toujours en filigrane, dans tout ce que tu écris. Peut-être que tu pourrais écrire un texte sur la manie?» C'est donc comme cela que ce travail a débuté.

LM: Vous savez, ce que j'apprécie beaucoup en vous écoutant, c'est qu'on vous sent toujours en mouvement. Je le dis parce que c'est ce qui nous porte. À votre façon, vous donnez de l'espoir. On sent vraiment qu'il n'y a rien d'établi pour vous, qu'il y a beaucoup de portes à ouvrir. On vous regarde aller, on sent l'analyste en vous, la femme curieuse, la femme qui cherche à comprendre en sachant qu'il va toujours y avoir quelque chose à comprendre. Merci beaucoup de vous être prêtée à l'entrevue. Vous nous donnez le goût de continuer de vous lire!

CC: Merci de m'avoir fait cette proposition d'échange. C'était un plaisir!

\section{Notes}

1. Cette entrevue - également disponible en ligne, sur notre site web - a été réalisée le 18 octobre 2015, à Québec. Catherine Chabert avait été invitée par la Société psychanalytique de Québec à l'occasion d'une journée clinique intitulée L'amour de la différence.

2. Journée clinique organisée par la Société psychanalytique de Québec. 